\title{
Uniqueness Problems about Entire Functions with Their Difference Operator Sharing Sets
}

\author{
Fan Niu, ${ }^{1}$ Jianming Qi ${ }^{1},{ }^{1}$ and Zhiyong $Z_{h o u}{ }^{2}$ \\ ${ }^{1}$ Business College, Shanghai Dianji University, Shanghai 200240, China \\ ${ }^{2}$ School of Design and Art, Shanghai Dianji University, Shanghai 200240, China \\ Correspondence should be addressed to Jianming Qi; qijianmingdaxia@163.com
}

Received 6 January 2020; Accepted 11 April 2020; Published 5 November 2020

Academic Editor: Sei-Ichiro Ueki

Copyright $(2020$ Fan Niu et al. This is an open access article distributed under the Creative Commons Attribution License, which permits unrestricted use, distribution, and reproduction in any medium, provided the original work is properly cited.

In this paper, we study the uniqueness questions of finite order transcendental entire functions and their difference operators sharing a set consisting of two distinct entire functions of finite smaller order. Our results in this paper improve the corresponding results from Liu (2009) and $\mathrm{Li}(2012)$.

\section{Introduction and Main Results}

Before proceeding, we spare the reader for a moment and assume some familiarity with the basics of Nevanlinna theory of meromorphic functions in $\mathbb{C}$ such as the first and second main theorems and the usual notations such as the characteristic function $T(r, f)$, the proximity function $m(r, f)$, and the counting function $N(r, f) . S(r, f)$ denotes any quantity satisfying $S(r, f)=o(T(r, f))$ as $r \longrightarrow \infty$, except possibly on a set of finite logarithmic measure not necessarily the same at each occurrence, see e.g., [1-3].

Let $f$ be a meromorphic functions on $\mathbb{C}$. Here, the order $\rho(f)$ is defined by

$$
\rho(f)=\limsup _{\mathrm{r} \longrightarrow \infty} \frac{\log T(r, f)}{\log r},
$$

and the exponent of convergence of zeros $\lambda(f)$ is defined by

$$
\lambda(f)=\limsup _{\mathrm{r} \longrightarrow \infty} \frac{\log N(r,(1 / f))}{\log r} .
$$

For a given $a \in \widehat{\mathbb{C}}=\mathbb{C} \cup\{\infty\}$, we say that two meromorphic functions $f$ and $g$ share $a \mathrm{CM}$ (counting multiplicities) when $f$ and $g$ have the same $a$-points. Let $S$ be a finite set of some entire functions and $f$ an entire function. Then, a set $E_{f}(S)$ is defined as

$$
E_{f}(S)=\bigcup_{a \in S}\{z \mid f(z)-a=0 \text {, counting multiplicities }\} .
$$

Assume that $g$ is another entire function. We say that $f$ and $g$ share a set $S$, counting multiplicities (CM), provided that $E_{f}(S)=E_{g}(S)$.

The uniqueness theory of meromorphic functions sharing sets generalizes that on sharing values and generally is more difficult. If meromorphic functions share a general set, it is not easy to determine these functions. In 1999, Li and Yang [4] deduced that if $E_{f}(S)=E_{f}^{\prime}(S)$ with $S$ contain two distinct constants, then $f$ must have special forms. Fang and Zalcman [5] used the theory of normal family to solve the above problem by proving that there exists a finite set $S$ containing three distinct elements such that if $E_{f}(S)=E_{f}^{\prime}(S)$, then $f=f^{\prime}$.

Recently years, Nevanlinna characteristic of $f(z+c)$, the value distribution theory for difference analogue, Nevanlinna theory of the difference operator, and the difference analogue of the lemma on the logarithmic derivative had been built, see e.g., [1-4, 6-14]. For meromorphic functions $f(z)$, we define its shift by $f_{c}(z)=f(z+c)$ and its difference operators by 


$$
\begin{aligned}
& \Delta_{c} f=f(z+c)-f(z), \\
& \Delta_{c}^{n} f=\Delta_{c}^{n-1}\left(\Delta_{c} f(z)\right), \quad n \in \mathbb{N}, n \geq 2 .
\end{aligned}
$$

By Nevanlinna theory of the difference operator, a natural question to ask whether the derivative $f^{\prime}$ can be replaced by the difference operator $\Delta_{c} f(z)=f(z+$ c) $-f(z)$ in the above question?

In 2009, Liu [8] investigated the above question and proved the following result.

Theorem 1. Let a be a nonzero complex number and $f$ be a transcendental entire function with finite order. If $f$ and $\Delta_{c} f$ share $\{a,-a\} C M$, then $\Delta_{c} f(z)=f(z)$ for all $z \in \mathbb{C}$.

In 2012, from Theorem 1, considering the constant in set is replaced by the function, $\mathrm{Li}$ [9] proved the following.

Theorem 2. If $a$ and $b$ are two distinct entire functions, then $f$ is a nonconstant entire function whose $\rho(f) \neq 1$ and $\lambda(f)<\rho(f)<\infty$ such that $\rho(a)<\rho(f)$ and $\rho(b)<\rho(f)$. If $f$ and $\Delta_{c} f$ share $\{a, b\} C M$, then $f(z)=\Delta_{c} f(z)$ for all $z \in \mathbb{C}$. follows.

After studying Theorem 2, we propose some questions as

Question 1: from Theorem 2, the condition $\rho(f) \neq 1$ seems more stronger. So, one may ask whether it can be weakened or moved?

Question 2: what will happen if the shift $\Delta_{c} f(z)$ be replaced by $\Delta_{c}^{n} f(z)(n \geq 2)$ in Theorem 2?

Fortunately, we have recently given a positive answer for Question 1 (see [14]). In this work, we also discuss the above problems and especially for Question 2. Finally, we derive the following results.

Theorem 3. Suppose that $a$ and $b$ are two distinct entire functions and $f$ is a nonconstant entire function of finite order with $\lambda(f)<\rho(f)<\infty$ such that $\rho(a)<\rho(f)$ and $\rho(b)<\rho(f)$. If $f$ and $\Delta_{c}^{2} f$ share $\{a, b\} C M$, then $f(z)$ must take one of the following conclusions:

(1) $f(z)=A e^{\mu z}$, where $A$ and $\mu$ are two nonzero constants satisfying $e^{\mu c}=2$. Furthermore, $f(z)=$ $\Delta_{c} f(z)$.

(2) $f(z)=H(z) e^{A z}$. Here, $H(z)$ is an entire function and $\lambda(f)=\rho(H)<1$.

Using the same method, we improve the above result from the shift $\Delta_{c} f(z)$ to $\Delta_{c}^{n} f(z)(n \geq 3)$ in above theorem and obtain the following result.

Theorem 4. Suppose that $a$ and $b$ are two distinct entire functions and $f$ is a nonconstant entire function of finite order with $\lambda(f)<\rho(f)<\infty$ such that $\rho(a)<\rho(f)$ and $\rho(b)<\rho(f)$. If $f$ and $\Delta_{c}^{n} f(n \geq 3)$ share $\{a, b\} C M$, then $f(z)=H(z) e^{A z}$, where $H(z)$ is an entire function such that $\lambda(f)=\rho(H)<1$.

\section{Some Lemmas}

We will introduce some lemmas for the proofs of our theorems in this section.

Lemma 1 (see [15]). Let $f$ be a meromorphic function of finite order and let $\omega_{1}$ and $\omega_{2}$ be two arbitrary complex numbers such that $\omega_{1} \neq \omega_{2}$. Assume that $\sigma$ is the order of $f$, then for each $\epsilon>0$, we have

$$
m\left(r, \frac{f\left(z+\omega_{1}\right)}{f\left(z+\omega_{2}\right)}\right)=O\left(r^{\sigma-1+\epsilon}\right) .
$$

Lemma 2 (see [16]). Let $g$ be a function transcendental and meromorphic in the plane with order less than 1. Set $h>0$. Then, there exists an $\epsilon$-set $E$ such that

$$
\frac{g(z+\omega)}{g(z)} \longrightarrow 1, \quad \text { when } z \longrightarrow \infty \text { in } \mathbb{C} \backslash E,
$$

uniformly in $\omega$ for $|\omega| \leq h$.

Lemma 3 (see [3]). Suppose that $f_{1}(z), f_{2}(z), \ldots$, $f_{n}(z)(n \geq 2)$ are meromorphic functions and $g_{1}(z)$, $g_{2}(z), \ldots, g_{n}(z)$ are entire functions satisfying the following conditions:

(1) $\sum_{j=1}^{n} f_{j}(z) e^{g_{j}(z)}=0$.

(2) $g_{j}(z)-g_{k}(z)$ are not constants for $1 \leq j<k \leq n$.

(3) For $1 \leq j \leq n, 1 \leq h<k \leq n, T\left(r, f_{j}\right)=o\left(T\left(r, e^{g_{h}-g_{k}}\right)\right)$, $(r \longrightarrow \infty, r \notin E)$. Then, $f_{j}(z)=0,(j=1,2, \ldots, n)$.

\section{Proof of Theorems}

Proof of Theorem 1. Due to $f$ and $\Delta_{c}^{2} f$ share $\{a, b\}$ CM, so we set

$$
\frac{\left(\Delta_{c}^{2} f-a\right)\left(\Delta_{c}^{2} f-b\right)}{(f-a)(f-b)}=e^{Q},
$$

where $Q$ is an entire function. And then it follows from (7) and $\max \{\rho(a), \rho(b)\}<\rho(f)<\infty$ that $Q$ is a polynomial.

Using Hadamard Factorization Theorem, we assume that $f(z)=h(z) e^{P(z)}$, where $h(\equiv 0)$ is an entire function and $P$ is a polynomial which satisfied

$$
\lambda(f)=\rho(h)<\rho(f)=\operatorname{deg}(P) .
$$

So,

$$
\begin{aligned}
\Delta_{c}^{2} f= & f(z+2 c)-2 f(z+c)+f(z)=(h(z+2 c) \\
& -2 h(z+c)+h(z)) e^{P(z)} .
\end{aligned}
$$


Put the forms of $f$ and $\Delta_{c}^{2} f$ into (7) to yield

$$
\begin{array}{r}
\left\{\left[h(z+2 c) e^{P(z+2 c)-P(z)}-2 h(z+c) e^{P(z+c)-P(z)}+h(z)\right] e^{P(z)}-a(z)\right\} \\
\left\{\left[h(z+2 c) e^{P(z+2 c)-P(z)}-2 h(z+c) e^{P(z+c)-P(z)}+h(z)\right] e^{P(z)}-b(z)\right\} \\
=\left(h(z) e^{P(z)}-a(z)\right)\left(h(z) e^{P(z)}-b(z)\right) e^{Q(z)} .
\end{array}
$$

Take $w_{1}=h(z+2 c) e^{P(z+2 c)-P(z)}-2 h(z+c) e^{P(z+c)-P(z)}+$ $h(z)$. We assume that $w_{1}=0$. Then,

$$
h(z+2 c) e^{P(z+2 c)}-2 h(z+c) e^{P(z+c)}+h(z) e^{p(z)}=0 .
$$

By Lemma 3, if $p(z+2 c)-p(z), p(z+c)-p(z)$, and $p(z+2 c)-p(z+c)$ are not constants, then $h(z)=0$, a contradiction.

So, $p(z+2 c)-p(z)=a_{1}, \quad p(z+c)-p(z)=b_{1}$, and $p(z+2 c)-p(z+c)=c_{1}$, (where $a_{1}, b_{1}$, and $c_{1}$ are three constants).

We can get $p^{\prime}(z+2 c)-p^{\prime}(z)=0, \quad p^{\prime}(z+c)-p^{\prime}$ $(z)=0$, and $p^{\prime}(z+2 c)-p^{\prime}(z+c)=0$.

Hence, $p(z)$ is a periodic function. We also know $p(z)$ is a polynomial. So, we get $p(z)=A z+B$ (where $A$ and $B$ are two constants, and $A \neq 0)$. So, we obtain $f(z)=H(z) e^{A z}$. Here, $H(z)$ is an entire function and $\lambda(f)=\rho(H)<1$.

If $w_{1} \neq 0$ below, obviously, $w_{1}$ is a small function of $e^{P}$. Rewrite (10) as

$$
e^{Q}=\frac{w_{1}^{2}\left[e^{P}-\left(a / w_{1}\right)\right]\left[e^{P}-\left(b / w_{1}\right)\right]}{h^{2}\left[e^{P}-(a / h)\right]\left[e^{P}-(b / h)\right]} .
$$

Note that $a \neq b$. Without loss of generality, we set $a \neq 0$. Suppose that $z_{0}$ is a zero of $e^{P}-(a / h)$, but not a zero of $w_{1}$. From (12), we may easily obtain that $z_{0}$ is a zero of $e^{P}-$ $\left(a / w_{1}\right)$ or $e^{P}-\left(b / w_{1}\right)$. We denote by $N_{1}\left(r, e^{P}\right)$ the reduced counting function of those common zeros of $e^{P}-(a / h)$ and $e^{P(z)}-\left(a / w_{1}\right)$. Similarly, we also denote $N_{2}\left(r, e^{P}\right)$ the reduced counting function of those common zeros of $e^{P}-$ $(a / h)$ and $e^{P}-\left(b / w_{1}\right)$. Then,

$$
\begin{aligned}
T\left(r, e^{P}\right) & =\bar{N}\left(r, \frac{1}{e^{P(z)}-(a / h)}\right)+S\left(r, e^{P}\right) \\
& =N_{1}\left(r, e^{P}\right)+N_{2}\left(r, e^{P}\right)+S\left(r, e^{P}\right),
\end{aligned}
$$

which implies that either $N_{1}\left(r, e^{P}\right) \neq S\left(r, e^{P}\right)$ or $N_{2}\left(r, e^{P}\right) \neq S\left(r, e^{P}\right)$. We distinguish the two cases as follows:

Case 1: $N_{1}\left(r, e^{P}\right) \neq S\left(r, e^{P}\right)$.

We may assume that $a_{0}$ is a common zero of $e^{P}-(a / h)$ and $e^{P}-\left(a / w_{1}\right)$. It is obvious that $a_{0}$ is a zero of $(a / h)-\left(a / w_{1}\right)$. If $(a / h)-\left(a / w_{1}\right)=0$, then

$$
\begin{aligned}
S\left(r, e^{P}\right) & \neq N_{1}\left(r, e^{P}\right) \leq N\left(r, \frac{1}{(a / h)-\left(a / w_{1}\right)}\right) \\
& \leq T\left(r, \frac{a}{h}-\frac{a}{w_{1}}\right)=S\left(r, e^{P}\right),
\end{aligned}
$$

a contradiction. Hence,

$$
h=w_{1} \text {. }
$$

It deduces

$$
2 \frac{h(z+c)}{h(z+2 c)}=e^{P(z+2 c)-P(z+c)}
$$

By Lemma 1 , for any $\epsilon>0$,

$$
\begin{aligned}
m\left(r, e^{P(z+2 c)-P(z+c)}\right) & =m\left(r, \frac{h(z+c)}{h(z+2 c)}\right)+O(1) \\
& =O\left(r^{\rho(h)-1+\epsilon}\right)+O(1)
\end{aligned}
$$

We also get $m\left(r, e^{P(z+2 c)-P(z+c)}\right)=[A+o(1)] r^{\rho(f)-1}$, where $A$ is a fixed positive constant.

If $\rho(f)>1$, using $\rho(f)>\rho(h)$ and the above estimates of $m\left(r, e^{P(z+2 c)-P(z+c)}\right)$, It easily gets a contradiction. So, $\rho(f) \leq 1$, this means that $e^{P(z+2 c)-P(z+c)}$ is a nonzero constant $c_{0}$. Then, (16) changes to

$$
2 \frac{h(z+c)}{h(z+2 c)}=c_{0}
$$

Also, noting that $1 \geq \rho(f)>\rho(h)$. Then, by Lemma 2, we get that there exists an $\epsilon$-set $E$, as $z \notin E$ and $|z| \longrightarrow \infty$ such that

$$
\frac{h(z+c)}{h(z+2 c)} \longrightarrow 1
$$

So, $c_{0}=2$ and $h(z+c)=h(z+2 c)$, and this also means that $h$ is a periodic function. If $h$ is a nonconstant function, then $\rho(h) \geq 1$, a contradiction. Therefore, $h$ is a constant. Noting that $\operatorname{deg}(P)=\rho(f) \leq 1$ and $f$ is a nonconstant entire function. Then, $\operatorname{deg}(P)=1$. Thus, we may set $f=A e^{\mu z}$, where $A$ and $\mu$ are two nonzero constants.

Using the assumption of Case 1 , one has $f-a$ and $\Delta_{c} f-a$ as common zeros, which are not zeros of $a$. Suppose that $\alpha_{0}$ is a common zero of $f-a$ and $\Delta_{c}^{2} f-a$ 
and not a zero of $a$. Then, $z_{0}$ is a zero of $\Delta_{c}^{2} f-f$. Moreover,

$$
f\left(z_{0}+2 c\right)-2 f\left(z_{0}+c\right)=0
$$

this implies that $e^{\mu c}=2$. Finally, we deduce $\Delta_{c} f=f$, which is the desired result.

Case 2: $N_{2}\left(r, e^{P}\right) \neq S\left(r, e^{P}\right)$.

Suppose $b_{0}$ which is a common zero of $e^{P}-(a / h)$ and $e^{P}-\left(b / w_{1}\right)$. Then, it is obvious that $b_{0}$ is a zero of $(a / h)-\left(b / w_{1}\right)$. If $(a / h)-\left(b / w_{1}\right) \neq 0$, then

$$
\begin{aligned}
S\left(r, e^{P}\right) & \neq N_{2}\left(r, e^{P}\right) \leq N\left(r, \frac{1}{(a / h)-\left(b / w_{1}\right)}\right) \\
& \leq T\left(r, \frac{a}{h}-\frac{b}{w_{1}}\right)=S\left(r, e^{P}\right),
\end{aligned}
$$

a contradiction. Hence,

$$
\frac{a}{h}-\frac{b}{w_{1}}=0
$$

If $b=0$, then $(a / h)=0$, a contradiction. Thus, $b=0$. We may set that $c_{0}$ is a zero of $e^{P}-(b / h)$, but not a zero of $w_{1}$. It follows from (12) that $c_{0}$ is a zero of $e^{P}-$ $\left(a / w_{1}\right)$ or $e^{P}-\left(b / w_{1}\right)$. We take by $N_{3}\left(r, e^{P}\right)$ the reduced counting function of those common zeros of $e^{P}-(b / h)$ and $e^{P}-\left(a / w_{1}\right)$. Similarly, we denote by $N_{4}\left(r, e^{P}\right)$ the reduced counting function of those common zeros of $e^{P}-(b / h)$ and $e^{P}-\left(b / w_{1}\right)$. We obtain

$$
\begin{aligned}
T\left(r, e^{P}\right)= & \bar{N}\left(r, \frac{1}{e^{P}-(b / h)}\right)+S\left(r, e^{P}\right)=N_{3}\left(r, e^{P}\right) \\
& +N_{4}\left(r, e^{P}\right)+S\left(r, e^{P}\right) .
\end{aligned}
$$

It implies that either $N_{3}\left(r, e^{P}\right) \neq S\left(r, e^{P}\right)$ or $N_{4}$ $\left(r, e^{P}\right) \neq S\left(r, e^{P}\right)$. If $N_{4}\left(r, e^{P}\right) \neq S\left(r, e^{P}\right)$, likewise with Case 1 , we deduce the desired result. Hence, we set that $N_{3}\left(r, e^{P}\right) \neq S\left(r, e^{P}\right)$ below. Similarly with Case 2 , we also get that

$$
\frac{b}{h}-\frac{a}{w_{1}}=0
$$

Combining (22) with (24), we deduce that

$$
a^{2}=b^{2} \text {. }
$$

Note that $a \neq b$. Thus, $a=-b$. Again using (24), we have $w_{1}=-h$. We rewrite it as

$$
h(z+2 c) e^{P(z+2 c)-P(z)}-2 h(z+c) e^{P(z+c)-P(z)}+2 h(z)=0 .
$$

Then,

$$
h(z+2 c) e^{P(z+2 c)}-2 h(z+c) e^{P(z+c)}+2 h(z) e^{P(z)}=0 .
$$

By Lemma 3, if $p(z+2 c)-p(z), p(z+c)-p(z)$, and $p(z+2 c)-p(z+c)$ are not constants, then $h(z)=0$; this is a contradiction.

So, $p(z+2 c)-p(z)=a_{1}, \quad p(z+c)-p(z)=b_{1}$, and $p(z+2 c)-p(z+c)=c_{1}$ (where $a_{1}, b_{1}$, and $c_{1}$ are three constants).

We can get $p^{\prime}(z+2 c)-p^{\prime}(z)=0, \quad p^{\prime}(z+c)-p^{\prime}$ $(z)=0$, and $p^{\prime}(z+2 c)-p^{\prime}(z+c)=0$.

Hence, $p(z)$ is a periodic function. We also know $p(z)$ is a polynomial. So, we get $p(z)=A z+B$ (where $A$ and $B$ are two constants, and $A \neq 0)$. So, we obtain $f(z)=H(z) e^{A z}$. Here, $H(z)$ is an entire function and $\lambda(f)=\rho(H)<1$.

Therefore, the proof of the main Theorem 3 is finished.

Proof of Theorem 2. Note that $f$ and $\Delta_{c}^{n} f$ share $\{a, b\}$ CM. So, we also set

$$
\frac{\left(\Delta_{c}^{n} f-a\right)\left(\Delta_{c}^{n} f-b\right)}{(f-a)(f-b)}=e^{Q},
$$

where $Q$ is an entire function. Furthermore, it deduces from (28) and $\max \{\rho(a), \rho(b)\}<\rho(f)<\infty$ that $Q$ is a polynomial.

Using Hadamard Factorization Theorem, we assume that $f(z)=h(z) e^{P(z)}$, where $h(\neq 0)$ is an entire function and $P$ is a polynomial satisfying

$$
\lambda(f)=\rho(h)<\rho(f)=\operatorname{deg}(P) .
$$

Then,

$$
\begin{aligned}
\Delta_{c}^{n} f= & f(z+n c)+b_{n-1} f(z+(n-1) c) \\
& +\cdots+b_{1} f(z+c)+b_{0} f(z) \\
= & h(z+n c) e^{p(z+n c)}+b_{n-1} h(z \\
& +(n-1) c) e^{p(z+(n-1) c)}+\cdots+b_{0} h(z) e^{p(z)},
\end{aligned}
$$

where $b_{0}, b_{1}, \ldots, b_{n-1}$ are constants. Substituting the forms of $f$ and $\Delta_{c}^{n} f$ into (28) yields

$$
\begin{aligned}
& \left\{\left[h(z+n c) e^{p(z+n c)-p(z)}+b_{n-1} h(z+(n-1) c) e^{p(z+(n-1) c)-p(z)}+\cdots+b_{0} h(z)\right] e^{P(z)}-a(z)\right\}, \\
& \left\{\left[h(z+n c) e^{p(z+n c)-p(z)}+b_{n-1} h(z+(n-1) c) e^{p(z+(n-1) c)-p(z)}+\cdots+b_{0} h(z)\right] e^{P(z)}-b(z)\right\} \\
& \quad=\left(h(z) e^{P(z)}-a(z)\right)\left(h(z) e^{P(z)}-b(z)\right) e^{Q(z)}
\end{aligned}
$$


Set $\quad w_{1}=h(z+n c) e^{p(z+n c)-p(z)}+b_{n-1} h(z+\quad(n-1) c)$ $e^{p(z+(n-1) c)-p(z)}+\cdots+b_{0} h(z)$. Suppose that $w_{1}=0$. Then,

$$
\begin{aligned}
h(z+n c) e^{p(z+n c)} & +b_{n-1} h(z+(n-1) c) e^{p(z+(n-1) c)} \\
& +\cdots+b_{0} h(z) e^{p(z)} 0 .
\end{aligned}
$$

By Lemma 3, if $p(z+n c)-p(z), p(z+(n-1) c)-$ $p(z), \ldots, p(z+c)-p(z)$ are not constants, then $h(z)=0$; a contradiction.

So, $\quad p(z+n c)-p(z)=a_{n}, \quad p(z+(n-1) c)-p(z)=$ $a_{n-1}$, and $p(z+c)-p(z)=a_{1}$ (where $a_{n}, a_{n-1}, \ldots, a_{1}$ are three constants).

We can get $p^{\prime}(z+n c)-p^{\prime}(z)=0, p^{\prime}(z+(n-1) c)-$ $p^{\prime}(z)=0$, and $p^{\prime}(z+c)-p^{\prime}(z)=0$.

Hence, $p(z)$ is a periodic function. We also know $p(z)$ is a polynomial. So, we get $p(z)=A z+B$ (where $A$ and $B$ are two constants, and $A \neq 0)$. So, we obtain $f(z)=H(z) e^{A z}$. Here, $H(z)$ is an entire function and $\lambda(f)=\rho(H)<1$.

If $w_{1} \neq 0$ below, obviously, $w_{1}$ is a small function of $e^{P}$. Rewrite (31) as

$$
e^{Q}=\frac{w_{1}^{2}\left[e^{P}-\left(a / w_{1}\right)\right]\left[e^{P}-\left(b / w_{1}\right)\right]}{h^{2}\left[e^{P}-(a / h)\right]\left[e^{P}-(b / h)\right]} .
$$

Due to $a \neq b$, without loss of generality, we set $a \neq 0$. Assume that $z_{0}$ is a zero of $e^{P}-(a / h)$, but not a zero of $w_{1}$. It deduces from (33) that $z_{0}$ is a zero of $e^{P}-\left(a / w_{1}\right)$ or $e^{P}-\left(b / w_{1}\right)$. We also take $N_{1}\left(r, e^{P}\right)$ the reduced counting function of those common zeros of $e^{P}-(a / h)$ and $e^{P}-\left(a / w_{1}\right)$. Likewise, we denote by $N_{2}\left(r, e^{P}\right)$ the reduced counting function of those common zeros of $e^{P}-(a / h)$ and $e^{P}-\left(b / w_{1}\right)$. Then,

$$
\begin{aligned}
T\left(r, e^{P}\right)= & \bar{N}\left(r, \frac{1}{e^{P(z)}-(a / h)}\right)+S\left(r, e^{P}\right)=N_{1}\left(r, e^{P}\right) \\
& +N_{2}\left(r, e^{P}\right)+S\left(r, e^{P}\right),
\end{aligned}
$$

this implies that either $N_{1}\left(r, e^{P}\right) \neq S\left(r, e^{P}\right)$ or $N_{2}$ $\left(r, e^{P}\right) \neq S\left(r, e^{P}\right)$. We may distinguish the following two cases.

Case 1: $N_{1}\left(r, e^{P}\right) \neq S\left(r, e^{P}\right)$.

We set $a_{0}$ a common zero of $e^{P}-(a / h)$ and $e^{P}-\left(a / w_{1}\right)$. Then, it is obvious that $a_{0}$ is a zero of $(a / h)-\left(a / w_{1}\right)$. If $(a / h)-\left(a / w_{1}\right) \neq 0$, then

$$
\begin{aligned}
S\left(r, e^{P}\right) & \neq N_{1}\left(r, e^{P}\right) \leq N\left(r, \frac{1}{(a / h)-\left(a / w_{1}\right)}\right) \\
& \leq T\left(r, \frac{a}{h}-\frac{a}{w_{1}}\right)=S\left(r, e^{P}\right),
\end{aligned}
$$

a contradiction. Hence,

$$
h=w_{1} \text {. }
$$

It leads to

$$
\begin{aligned}
h(z+n c) e^{p(z+n c)} & +b_{n-1} h(z+(n-1) c) e^{p(z+(n-1) c)} \\
& +\cdots+\left(b_{0}-1\right) h(z) e^{p(z)}=0
\end{aligned}
$$

By Lemma 3, if $p(z+n c)-p(z), p(z+(n-1) c)-$ $p(z), \ldots p(z+c)-p(z)$, are not constants, then $h(z)=0$, a contradiction.

So, $\quad p(z+n c)-p(z)=a_{n}, \quad p(z+(n-1) c)-p(z)=$ $a_{n-1}, \ldots, p(z+c)-p(z)=a_{1}$, (where $a_{n}, a_{n-1}, a_{1}$ are three constants).

We can get $p^{\prime}(z+n c)-p^{\prime}(z)=0, p^{\prime}(z+(n-1) c)-$ $p^{\prime}(z)=0, \ldots, p^{\prime}(z+c)-p^{\prime}(z)=0$.

Here, $p(z)$ is a periodic function. We also know $p(z)$ is a polynomial. So, we get $p(z)=A z+B$ (where $A$ and $B$ are two constants, and $A \neq 0$ ). Finally, we get $f(z)=H(z) e^{A z}$, where $H(z)$ is an entire function and $\lambda(f)=\rho(H)<1$.

Case 2: $N_{2}\left(r, e^{P}\right) \neq S\left(r, e^{P}\right)$.

Suppose $b_{0}$ is a common zero of $e^{P}-(a / h)$ and $e^{P}-\left(b / w_{1}\right)$. Then, it is easy to see that $b_{0}$ is a zero of $(a / h)-\left(b / w_{1}\right)$. If $(a / h)-\left(b / w_{1}\right) \neq 0$, then

$$
\begin{aligned}
S\left(r, e^{P}\right) & \neq N_{2}\left(r, e^{P}\right) \leq N\left(r, \frac{1}{(a / h)-\left(b / w_{1}\right)}\right) \\
& \leq T\left(r, \frac{a}{h}-\frac{b}{w_{1}}\right)=S\left(r, e^{P}\right),
\end{aligned}
$$

a contradiction. Thus,

$$
\frac{a}{h}-\frac{b}{w_{1}}=0 .
$$

If $b=0$, then $(a / h)=0$, a contradiction. Thus, $b \neq 0$.

We assume that $c_{0}$ is a zero of $e^{P}-(b / h)$, but not a zero of $w_{1}$. It deduces from (33) that $c_{0}$ is a zero of $e^{P}-$ $\left(a / w_{1}\right)$ or $e^{P}-\left(b / w_{1}\right)$. We take by $N_{3}\left(r, e^{P}\right)$ the reduced counting function of those common zeros of $e^{P}-(b / h)$ and $e^{P}-\left(a / w_{1}\right)$. Similarly, we denote by $N_{4}\left(r, e^{P}\right)$ the reduced counting function of those common zeros of $e^{P}-(b / h)$ and $e^{P}-\left(b / w_{1}\right)$. Then,

$$
\begin{aligned}
T\left(r, e^{P}\right)= & \bar{N}\left(r, \frac{1}{e^{P}-(b / h)}\right)+S\left(r, e^{P}\right)=N_{3}\left(r, e^{P}\right)+N_{4}\left(r, e^{P}\right) \\
& +S\left(r, e^{P}\right),
\end{aligned}
$$


and this implies that either $N_{3}\left(r, e^{P}\right) \neq S\left(r, e^{P}\right)$ or $N_{4}\left(r, e^{P}\right) \neq S\left(r, e^{P}\right)$. If $N_{4}\left(r, e^{P}\right) \neq S\left(r, e^{P}\right)$. Similarly, as the same way in Case 1, we get the desired result. So, we assume that $N_{3}\left(r, e^{P}\right) \neq S\left(r, e^{P}\right)$ as follows. Similarly, as the way in Case 2 , we can get that

$$
\frac{b}{h}-\frac{a}{w_{1}}=0
$$

It follows from (39) and (41) that

$$
a^{2}=b^{2} \text {. }
$$

Note that $a \neq b$. Thus, $a=-b$. Again by (41), one has $w_{1}=-h$. We also rewrite it as

$$
\begin{aligned}
h(z+n c) e^{p(z+n c)} & +b_{n-1} h(z+(n-1) c) e^{p(z+(n-1) c)} \\
& +\cdots+\left(b_{0}+1\right) h(z) e^{p(z)}=0 .
\end{aligned}
$$

By Lemma 3, if $p(z+n c)-p(z), p(z+(n-1) c)-$ $p(z), \ldots, p(z+c)-p(z)$ are not constants, then $h(z)=0$, a contradiction.

So, $\quad p(z+n c)-p(z)=a_{n}, \quad p(z+(n-1) c)-p(z)=$ $a_{n-1}, \ldots, p(z+c)-p(z)=a_{1}$ (where $a_{n}, a_{n-1}, \ldots, a_{1}$ are three constants).

We also get $p^{\prime}(z+n c)-p^{\prime}(z)=0, p^{\prime}(z+(n-1) c)-$ $p^{\prime}(z)=0, \ldots, p^{\prime}(z+c)-p^{\prime}(z)=0$.

Hence, $p(z)$ is a periodic function. We also know $p(z)$ is a polynomial. So, we get $p(z)=A z+B$ (where $A$ and $B$ are two constants, and $A \neq 0)$. Finally, we get $f(z)=H(z) e^{A z}$. Here, $H(z)$ is an entire function and $\lambda(f)=\rho(H)<1$.

Proof of Theorem 4 is completed.

\section{Data Availability}

No data were used to support this study.

\section{Conflicts of Interest}

The authors declare that they have no conflicts of interest.

\section{Authors' Contributions}

All authors typed, read, and approved the final manuscript.

\section{Acknowledgments}

The work presented in this paper was supported by the Plateau Disciplines in Shanghai, Leading Academic Discipline Project of Shanghai Dianji University (16JCXK02), and Philosophy and Social Sciences Planning Project of the Ministry of Education (Grant no. 18YJC630120).

\section{References}

[1] W. K. Hayman, Meromorphic Functions, Clarendon Press, Oxford, UK, 1964.

[2] I. Laine, Nevanlinna Theory and Complex Differential Equations, Walter de Gruyter, Berlin, Germany, 1993.

[3] C. C. Yang and H. X. Yi, Uniqueness Theory of Meromorphic Functions, Science Press, Beijing, Germany, 2003.
[4] P. Li and C.-C. Yang, "Value sharing of an entire function and its derivatives," Journal of the Mathematical Society of Japan, vol. 51, no. 4, pp. 781-799, 1999.

[5] M. Fang and L. Zalcman, "Normal families and uniqueness theorems for entire functions," Journal of Mathematical Analysis and Applications, vol. 280, no. 2, pp. 273-283, 2003.

[6] R. G. Halburd and R. J. Korhonen, "Nevanlinna theory for the difference operators," Annales Academiae Scientiarum Fennicae Mathematica, vol. 31, no. 2, pp. 463-478, 2006.

[7] R. G. Halburd and R. J. Korhonen, "Difference analogue of the lemma on the logarithmic derivative with applications to difference equations," Journal of Mathematical Analysis and Applications, vol. 314, no. 2, pp. 477-487, 2006.

[8] K. Liu, "Meromorphic functions sharing a set with applications to difference equations," Journal of Mathematical Analysis and Applications, vol. 359, no. 1, pp. 384-393, 2009.

[9] X.-M. Li, "Entire functions sharing a finite set with their difference operators," Computational Methods and Function Theory, vol. 12, no. 1, pp. 307-328, 2012.

[10] S. Li and B. Q. Chen, "Results on meromorphic solutions of linear difference equations," Advances in Difference Equations, vol. 2012, pp. 1-7, Article ID 203, 2012.

[11] S. Li, D. Mei, and B. Q. Chen, "Uniqueness of entire functions sharing two values with their difference operators," Advances in Difference Equations, vol. 2017, pp. 1-9, Article ID 390, 2017.

[12] S. Li, D. Mei, and B. Q. Chen, "Meromorphic functions sharing small functions with their linear difference polynomials," Advances in Difference Equations, vol. 2013, pp. 1-6, Article ID 58, 2013.

[13] I. Laine and C.-C. Yang, "Clunie theorems for difference and $q$ -difference polynomials," Journal of the London Mathematical Society, vol. 76, no. 3, pp. 556-566, 2007.

[14] J. M. Qi, Y. F. Wang, and Y. Y. Gu, "A note on entire functions sharing a finite set with their difference operators," Advances in Difference Equations, vol. 2019, pp. 1-7, Article ID 114, 2019.

[15] Y.-M. Chiang and S.-J. Feng, "On the Nevanlinna characteristic of $f(z+\eta)$ and difference equations in the complex plane," The Ramanujan Journal, vol. 16, no. 1, pp. 105-129, 2008. 